\title{
ENUMERATION OF BRANCHED COVERINGS OF NONORIENTABLE SURFACES WITH CYCLIC BRANCH POINTS*
}

\author{
JIN HO KWAK ${ }^{\dagger}$, ALEXANDER MEDNYKH ${ }^{\ddagger}$, AND VALERY LISKOVETS ${ }^{\S}$
}

\begin{abstract}
In this paper, $n$-fold branched coverings of a closed nonorientable surface $\mathcal{S}$ of genus $p$ with $r \geq 1$ cyclic branch points (that is, such that all ramification points over them are of multiplicity $n$ ) are considered. The number $N_{p, r}(n)$ of such coverings up to equivalence is evaluated explicitly in a closed form (without using any complicated functions such as irreducible characters of the symmetric groups). The obtained formulas depend on the parity of $r$ and $n$. The method is based on some previous enumerative results and techniques for nonorientable surfaces. In particular, we generalize the approach developed for the counting of unbranched coverings of nonorientable surfaces and make use of the analytical method of roots-of-unity sums.
\end{abstract}

Key words. cyclic branch point, covering of a nonorientable surface, Ramanujan sum, von Sterneck function, fundamental group, permutation tuple, Hurwitz number

AMS subject classifications. 57M10, 20F34, 14H30

DOI. $10.1137 / \mathrm{S} 0895480103424043$

1. Introduction. Throughout this paper, a surface means a compact connected 2-manifold without boundary. Recall some known concepts from algebraic topology [16]. A continuous mapping $\rho: \mathcal{T} \rightarrow \mathcal{S}$ from a surface $\mathcal{T}$ onto $\mathcal{S}$ is called a branched covering of multiplicity $n$ if there exists a finite subset $B=\left\{b_{1}, \ldots, b_{r}\right\}$ of points in $\mathcal{S}$ such that the restriction of $\rho$ on $\mathcal{T}-\rho^{-1}(B),\left.\rho\right|_{\mathcal{T}-\rho^{-1}(B)}: \mathcal{T}-\rho^{-1}(B) \rightarrow \mathcal{S}-B$, is an $n$-fold ( $n$-sheeted) covering projection in the usual sense. The smallest subset $B$ of $\mathcal{T}$ which has this property is called the branch point set of $\rho$. At the neighborhood of each point $x \in \rho^{-1}(B)$, the projection $\rho$ is topologically equivalent to the complex map $z \mapsto z^{m}$ with some natural number $m$. Such an $x$ is called a ramification point of $\rho$, and $m$ is called the order of $x$. Denote by $s_{m}^{k}$ the number of ramification points of order $m$ of the mapping $\rho$ in the preimage $\rho^{-1}\left(b_{k}\right)$, where $k=1, \ldots, r$ and $m=1, \ldots, n$. We will call the $(r \times n)$-matrix $\sigma=\left(s_{m}^{k}\right)$ the ramification type of the covering $\rho$. For any $k,\left(1^{s_{1}^{k}} \cdots n^{s_{n}^{k}}\right)$ is a partition of $n$, that is, $\sum_{m} m s_{m}^{k}=n$.

Two branched coverings $\rho: \mathcal{T} \rightarrow \mathcal{S}$ and $\rho^{\prime}: \mathcal{T}^{\prime} \rightarrow \mathcal{S}$ are considered to be equivalent (or isomorphic) if there exists a homeomorphism $\eta: \mathcal{T}^{\prime} \rightarrow \mathcal{T}$ such that $\rho^{\prime}=\rho \circ \eta$.

The classical Hurwitz enumeration problem is to count nonequivalent $n$-fold coverings of $\mathcal{S}$ with a given ramification type $\sigma$. By now, only the nonorientable case for branched coverings remains open. The orientable case was, in principle, solved completely by Mednykh [18], as was the nonorientable case with unramified coverings [20]. The aim of the present work is to adjust the method of the latter article to coverings of nonorientable surfaces in the particular case when $B$ is nonempty, $s_{m}^{k}=0$ for $m<n$, and $s_{n}^{k}=1$ for all $k=1, \ldots, r$. In other words, we consider the case when

\footnotetext{
* Received by the editors March 10, 2003; accepted for publication (in revised form) January 6, 2005; published electronically October 31, 2005. This research was supported by $\mathrm{Com}^{2} \mathrm{MaC}-\mathrm{KOSEF}$. http://www.siam.org/journals/sidma/19-2/42404.html

${ }^{\dagger}$ Combinatorial and Computational Mathematics Center, Pohang University of Science and Technology, Pohang, 790-784, Korea (jinkwak@postech.ac.kr).

‡Institute of Mathematics, Novosibirsk State University, 630090, Novosibirsk, Russia (mednykh@ math.nsc.ru).

$\S$ Institute of Mathematics, National Academy of Sciences of Belarus, 220072, Minsk, Belarus (liskov@im.bas-net.by).
} 
every branch point is cyclic; i.e., it is lifted to a unique ramification point (so that the corresponding covering permutation is a full cycle of length $n$ ). Such a restriction simplifies the situation considerably, allowing elimination of irreducible characters of the symmetric groups in the formulas. Recently, this idea has been implemented successfully by two of the present authors to the cases of orientable surfaces [11]. The present work supplements this paper, extending its results to nonorientable surfaces. A special technique of counting the solutions of systems of linear congruences by sums of roots of unity (known also as Ramanujan's sums) is applied. See [11] for all necessary definitions, references, and additional explanations. For other useful information concerning branched coverings of nonorientable surfaces, see $[8,9,10]$.

2. Preliminary results. In what follows, $\mathcal{S}$ denotes a closed nonorientable surface of genus $p$. The set of branch points $B$ will be considered fixed. We denote by $\mathbf{S}_{n}$ the symmetric groups on $n$ symbols and by $[g]$ the cycle type of a permutation $g \in \mathbf{S}_{n}$, that is, $[g]=\left(1^{s_{1}} \cdots n^{s_{n}}\right)$ if $g$ consists of $s_{m}$ independent cycles of length $m, m=1, \ldots, n$. For cycle types (partitions of $n$ ) we adopt the usual notational agreement to drop empty parts $m^{0}$ and to write $m$ instead of $m^{1}$. In particular, $(n)$ denotes the partition of $n$ consisting of a sole part, $n$.

As follows from results of Hurwitz [4] and their subsequent generalizations, each covering $\rho$ of $\mathcal{S}$ with the ramification type $\sigma=\left(s_{m}^{k}\right)$ is uniquely determined by an ordered $(p+r)$-tuple of permutations of degree $n$,

$$
\left(a_{1}, \ldots, a_{p}, c_{1}, \ldots, c_{r}\right) \in \mathbf{S}_{n}^{p+r}=\underbrace{\mathbf{S}_{n} \times \mathbf{S}_{n} \times \cdots \times \mathbf{S}_{n}}_{p+r},
$$

which satisfy the relations

$$
\begin{gathered}
\prod_{j=1}^{p} a_{j}^{2} \prod_{k=1}^{r} c_{k}=\mathbb{1}, \\
{\left[c_{k}\right]=\left(1^{s_{1}^{k}} \cdots n^{s_{n}^{k}}\right), \quad k=1,2, \ldots, r,}
\end{gathered}
$$

and generate a transitive subgroup of $\mathbf{S}_{n}$. Here $\mathbb{1}=\mathbb{1}_{n}$ denotes the identity permutation. Tuples satisfying the last condition will be called transitive. Two coverings are equivalent if and only if the corresponding tuples are conjugate via a permutation from $\mathbf{S}_{n}$. The proof of these facts can be found, for example, in [2] or [8].

Denote by $\mathfrak{B}_{p, r, \sigma}(n)$ the set of all tuples (transitive or not) of form (1) satisfying $(2)$ and (3), and select in $\mathfrak{B}_{p, r, \sigma}(n)$ the subset $\mathfrak{T}_{p, r, \sigma}(n)$ of transitive tuples. We set $B_{p, r, \sigma}(n):=\left|\mathfrak{B}_{p, r, \sigma}(n)\right|$ and $T_{p, r, \sigma}(n):=\left|\mathfrak{T}_{p, r, \sigma}(n)\right|$, where the vertical bars denote the cardinality of the set.

2.1. A general formula. The following result based on general formulas in terms of the irreducible characters for the number of solutions of equations in groups is valid (see [7]; cf. also [3, 6, 11, 20]).

Proposition 2.1. The number $B_{p, r, \sigma}(n)$ of elements of the set $\mathfrak{B}_{p, r, \sigma}(n)$ is determined by the formula

$$
B_{p, r, \sigma}(n)=n ! \sum_{\lambda \in D_{n}}\left(\prod_{k=1}^{r} \frac{\chi_{s_{1}^{k} \cdots s_{n}^{k}}^{\lambda}}{1^{s_{1}^{k}} \cdot s_{1}^{k} ! \cdots n_{n}^{s_{n}^{k}} \cdot s_{n}^{k} !}\right)\left(\frac{n !}{f^{\lambda}}\right)^{p-2+r},
$$


where $\sigma=\left(s_{m}^{k}\right)$ is the ramification type, $D_{n}$ is the set of all irreducible representations of the group $\mathbf{S}_{n}, f^{\lambda}$ is the degree, and $\chi_{s_{1}^{k} \ldots s_{n}^{k}}^{\lambda}=\chi_{\left[c_{k}\right]}^{\lambda}$ is the character of permutations of the type $\left[c_{k}\right]=\left(1^{s_{1}^{k}} \cdots n^{s_{n}^{k}}\right)$ corresponding to the representation $\lambda$.

As noted, unlike branched coverings over orientable surfaces considered in [11], no general results have been obtained so far for the number of branched coverings over nonorientable surfaces. Therefore we use here special tools sufficient for the particular case under consideration, which generalize those given in [20] and [18]. Our task is facilitated partially by a somewhat similar problem considered in [13] (see also [14]) for a class of three-dimensional manifolds.

2.2. Cyclic branch points. In the case of cyclic branch points, $\sigma$ has a particular form:

$$
\left[c_{k}\right]=(n), \quad k=1,2, \ldots, r,
$$

that is, all $c_{k}$ are $n$-cycles. We denote by $T_{p, r}(n)$ the corresponding number of tuples, i.e., $T_{p, r}(n)=\left|\mathfrak{T}_{p, r,(n)^{r}}(n)\right|:=T_{p, r, \sigma}(n)$, where $\sigma$ is of form (5), i.e., $s_{n}^{k}=1$ and $s_{m}^{k}=0$ for $m<n$ and $k=1, \ldots, r$.

Our aim in this paper is to find the number of covering $N_{p, r}(n)$ up to equivalence, which coincides with the number of orbit of the symmetric group $\mathbf{S}_{n}$ acting by conjugation on the set $\mathfrak{T}_{p, r,(n)^{r}}(n)$. Notice also that $T_{p, r}(n) /(n-1) !$ is the number of subgroups of index $n$ of the corresponding fundamental group while $N_{p, r}(n)$ is the number of conjugacy classes of such subgroups. In the literature, $T_{p, r}(n)$ are also called the corresponding Hurwitz numbers.

TheOREM 2.2 (cf. [11]). For any $n, r \geq 1$, and $p \geq 0$, the number of tuples (1) satisfying conditions (2) and (5) is the following:

$$
T_{p, r}(n)=\frac{(n !)^{p-1+r}}{n^{r}} \sum_{s=0}^{n-1}(-1)^{s r}\left(\begin{array}{c}
n-1 \\
s
\end{array}\right)^{-(p-2+r)} .
$$

Proof. The presence of full cycles $c_{k}$ ensures transitivity, so that $\mathfrak{T}_{p, r,(n)^{r}}(n)=$ $\mathfrak{B}_{p, r,(n)^{r}}(n)$. This simplifies the enumeration considerably; in particular by Proposition 2.1 we have

$$
T_{p, r}(n)=n ! \sum_{\lambda \in D_{n}}\left(\frac{\chi_{(n)}^{\lambda}}{n}\right)^{r}\left(\frac{n !}{f^{\lambda}}\right)^{p-2+r} .
$$

Further we make use of the fact that characters $\chi^{\lambda}$ almost always vanish on the full cycle $(n)$. Namely,

$$
\chi_{(n)}^{\lambda}= \begin{cases}(-1)^{s} & \text { if } \lambda \vdash\left(1^{s} n-s\right), \quad 0 \leq s \leq n-1, \\ 0 & \text { otherwise }\end{cases}
$$

see, e.g., [5, Theorem 21.4] or [22, Example 7.67(a)]. Now, by the hook-length formula $[22,7.21 .6]$ we have

$$
f^{\lambda}=\frac{n !}{s ! n(n-s-1) !}=\left(\begin{array}{c}
n-1 \\
s
\end{array}\right) \quad \text { if } \quad \lambda \vdash\left(1^{s} n-s\right) .
$$

Substituting (8) and (9) into (7) we obtain (6). 
Due to formula (6), in the counting of permutation tuples for the case of cyclic branch points we have got rid of using characters.

Notice that in accordance with (6), $T_{p, r}(n)=0$ for odd $r$ and even $n$ since in this case a product of $r$ full cycles is an odd permutation; thus, equality (2) is impossible. However, we will not exclude this case from the subsequent consideration.

2.3. Calculations in the centralizer of a regular permutation. Since we need to count transitive permutation tuples up to conjugacy, we make use of enumerative Burnside's lemma. Accordingly we are interested in the automorphisms of tuples, that is, their centralizers. It is well known that each automorphism is a regular permutation. Hence, all permutations in such a tuple commute with this permutation $h$. Thus they belong to its centralizer $Z(h)$, which is of the form $\mathbb{Z}_{\ell} \prec \mathbf{S}_{m}$, where $\ell$ is the order of the automorphism. Now, our approach (going back to [12] and [17]) is to make necessary calculations in this wreath product so to take into account conditions (2) and (5).

Denote by $b^{g}$ the action of a permutation $g$ on an element $b$. Let us fix a regular permutation $h$ of degree $n$ and order $\ell(\ell m=n)$ which commutes with all permutations $a_{1}, a_{2}, \ldots, a_{p}$ and $c_{1}, c_{2}, \ldots, c_{r}$. Belonging to $Z(h) \cong \mathbb{Z}_{\ell}\left\langle\mathbf{S}_{m}\right.$, they can be written in the form

$$
a_{i}=\left(t_{1}^{i}, t_{2}^{i}, \ldots, t_{m}^{i} ; \hat{a}_{i}\right), \quad i=1,2, \ldots, p,
$$

and

$$
c_{k}=\left(x_{1}^{k}, x_{2}^{k}, \ldots, x_{m}^{k} ; \hat{c}_{k}\right), \quad k=1, \ldots, r,
$$

where all $\hat{a}_{i}$ and $\hat{c}_{k}$ belong to $\mathbf{S}_{m}$ and all $t_{j}^{i}$ and $x_{j}^{k}, j=1, \ldots, m$, belong to $\mathbb{Z}_{\ell}$. Now using the formulas of the multiplication of permutations in $\mathbb{Z}_{\ell} \imath \mathbf{S}_{m}$ described, say, in [14], we can represent (2) as the following system of congruences:

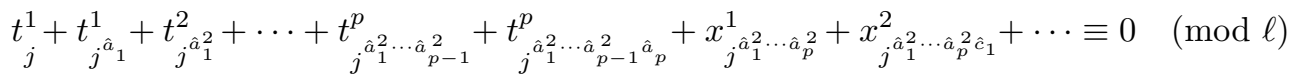

for $j=1, \ldots, m$ together with the equation

$$
\hat{a}_{1}^{2} \hat{a}_{2}^{2} \cdots \hat{a}_{p}^{2} \hat{c}_{1} \cdots \hat{c}_{r}=\mathbb{1}_{m}
$$

It is easy to see (see [18]) that in these terms, condition (5) is expressed as follows:

$$
\left(x_{1}^{k}+x_{2}^{k}+\cdots+x_{m}^{k}, \ell\right)=1, \quad k=1,2, \ldots, r, \quad r \geq 1,
$$

where again $($,$) denotes the greatest common divisor, and$

$$
\left[\hat{c}_{k}\right]=(m), \quad k=1,2, \ldots, r .
$$

Now we are interested in the number of solutions of the system of (10) and (12). This number proves (as we will see later) to be independent of a specific choice of the tuple $\left(a_{1}, \ldots, a_{p}, c_{1}, \ldots, c_{r}\right)$. More generally, let us consider an arbitrary $(2 p+r)$-tuple of permutations of degree $m,\left(\alpha_{1}, \beta_{1}, \ldots, \alpha_{p}, \beta_{p}, \gamma_{1}, \ldots, \gamma_{r}\right)$, where $\gamma_{1}, \ldots, \gamma_{r}$ are full cycles, and let $M=M_{p, r, m}(\ell)$ denote the number of solutions of the system (12) and (14) in $\mathbb{Z}_{\ell}$, where

$$
t_{j^{\alpha_{1}}}^{1}+t_{j^{\beta_{1}}}^{1}+\cdots+t_{j^{\alpha_{p}}}^{p}+t_{j^{\beta_{p}}}^{p}+x_{j^{\gamma_{1}}}^{1}+\cdots+x_{j^{\gamma_{r}}}^{r} \equiv 0 \quad(\bmod \ell), \quad j=1, \ldots, m .
$$


The following lemma is a crucial technical result of this work.

Lemma 2.3. For any tuple $\left(\alpha_{1}, \beta_{1}, \ldots, \alpha_{p}, \beta_{p}, \gamma_{1}, \ldots, \gamma_{r}\right) \in \mathbf{S}_{m}^{2 p+r}$, where all $\gamma_{i}$ are full cycles, the number of solutions of the system of (12) and (14) in $\mathbb{Z}_{\ell}$ is determined by the following formula:

$$
M_{p, r, m}(\ell)=\ell^{m(p-1+r)-r} \phi(\ell)^{r} \times \begin{cases}1 & \text { for } \ell \text { odd }, \\ 2 & \text { for } \ell \text { even, } r \text { even }, \\ 0 & \text { for } \ell \text { even }, r \text { odd }\end{cases}
$$

where $\phi(\ell)$ is the Euler function.

Proof. Generally we make use of the same technique as in the appendix of [13] (see also [20]). Denoting by $f_{j}$ the left-hand-side expressions of (14), we introduce the following polynomials of $z_{1}, \ldots, z_{m}$ :

$$
P\left(z_{1}, \ldots, z_{m}\right):=\sum_{\substack{\forall i, j, k \\\left(x_{1}^{k}+\cdots+x_{m}^{i}, x_{j}^{k} \leq \ell\right)=1}} \prod_{j=1}^{m} z_{j}^{f_{j}}
$$

Then the number of solutions of the system (12) and (14) modulo $\ell$ coincides with the sum of the coefficients of $P\left(z_{1}, \ldots, z_{m}\right)$, all indices of which are divisible by $\ell$, and, consequently, is given by the formula

$$
M=\frac{1}{\ell^{m}} \sum_{\substack{1 \leq \ell_{1} \leq \ell \\ 1 \leq \dot{\ell}_{m} \leq \ell}} P\left(\varepsilon^{\ell_{1}}, \ldots, \varepsilon^{\ell_{m}}\right),
$$

where $\varepsilon=\sqrt[\ell]{1}=\exp \frac{2 \pi i}{\ell}, i=\sqrt{-1}$.

Changing the order of the factors in (16) by applying $\alpha_{i}^{-1}, \beta_{i}^{-1}$, and $\gamma_{i}^{-1}$ to subscripts, one can represent $\prod_{j=1}^{m} z_{j}^{f_{j}}$ as follows:

$$
P\left(z_{1}, \ldots, z_{m}\right)=\sum_{\substack{1 \leq t_{j}^{i}, x_{j}^{k} \leq \ell \\\left(x_{1}^{k}+\cdots+x_{m}^{k}, \ell\right)=1}} \prod_{j=1}^{m}\left(z_{j^{\alpha_{1}^{-1}}}^{t_{j}^{1}} z_{j^{\beta_{1}^{-1}}}^{t_{j}^{1}} \cdots z_{j^{\alpha_{p}^{-1}}}^{t_{j}^{p}} z_{j^{\beta_{p}^{-1}}}^{t_{j}^{p}} z_{j^{\gamma_{1}^{-1}}}^{x_{1}^{1}} \cdots z_{j^{\gamma_{r}^{-1}}}^{x^{r}}\right)
$$

whence by elementary, although tedious, transformations,

$$
\begin{aligned}
& P\left(\varepsilon^{\ell_{1}}, \ldots, \varepsilon^{\ell_{m}}\right)
\end{aligned}
$$

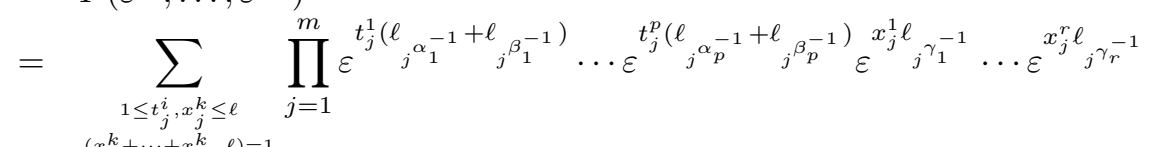

$$
\begin{aligned}
& \left(x_{1}^{k}+\cdots+x_{m}^{k}, \ell\right)=1
\end{aligned}
$$

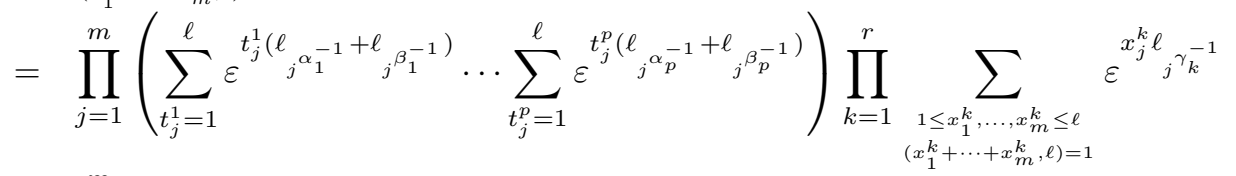

$$
\begin{aligned}
& =\prod_{j=1}^{m}\left[\ell \delta\left(0, \ell_{j_{1}^{\alpha_{1}^{-1}}}+\ell_{j^{\beta_{1}^{-1}}}\right) \cdots \ell \delta\left(0, \ell_{j^{\alpha_{p}^{-1}}}+\ell_{j^{\beta_{p}^{-1}}}\right)\right] \delta\left(\ell_{1}, \ell_{2}, \ldots, \ell_{m}\right) \Phi\left(\ell_{1}, \ell\right)^{r} \ell^{r(m-1)} .
\end{aligned}
$$


Here we use a multivariable $\delta$-function defined as follows:

$$
\delta(a, b, c, \ldots):= \begin{cases}1 & \text { if } a \equiv b \equiv c \equiv \ldots(\bmod \ell), \\ 0 & \text { otherwise }\end{cases}
$$

The last equality in (18) is based on the following claim.

CLAim 1. $\quad \Sigma:=\sum_{\substack{1 \leq x_{1}, \ldots, x_{m} \leq \ell \\\left(x_{1}+\ldots+x_{m}, \ell\right)=1}} \varepsilon^{x_{j} \ell_{j}}=\delta\left(\ell_{1}, \ldots, \ell_{m}\right) \ell^{m-1} \Phi\left(\ell_{1}, \ell\right)$, where $\Phi(u, \ell)$ is the von Sterneck function (known also as Ramanujan's sum): the sum of the primitive $\ell$ th roots of unity in the power $u$ :

$$
\Phi(u, \ell):=\sum_{x:(x, \ell)=1} \varepsilon^{x u} .
$$

In turn, Claim 1 relies on the following well-known identity.

Claim 2. For any two integers $a$ and $b$, we have $\sum_{x=1}^{\ell} \varepsilon^{x(a-b)}=\ell \delta(a, b)$.

We have

$$
\Sigma=\sum_{x_{1}=1}^{\ell} \varepsilon^{\left(\ell_{1}-\ell_{m}\right) x_{1}} \ldots \sum_{x_{m-1}=1}^{\ell} \varepsilon^{\left(\ell_{m-1}-\ell_{m}\right) x_{m-1}} \sum_{(x, \ell)=1} \varepsilon^{\ell_{m} x}=\delta\left(\ell_{1}, \ldots, \ell_{m}\right) \ell^{m-1} \Phi\left(\ell_{m}, \ell\right),
$$

where $x:=x_{1}+\cdots+x_{m}$. Besides, $\delta\left(\ell_{1}, \ldots, \ell_{m}\right) \Phi\left(\ell_{m}, \ell\right)=\delta\left(\ell_{1}, \ldots, \ell_{m}\right) \Phi\left(\ell_{1}, \ell\right)$ since both products vanish unless $\ell_{1}=\ell_{2}=\cdots=\ell_{m}$. These arguments prove Claim 1.

Return to the proof of Lemma 2.3. The factor $\delta\left(\ell_{1}, \ldots, \ell_{m}\right)$ in the last expression in (18) shows that the polynomial $P\left(\varepsilon^{\ell_{1}}, \ldots, \varepsilon^{\ell_{m}}\right)$ does not vanish only if all $\ell_{j}$ coincide,

$$
\ell_{1}=\cdots=\ell_{m}=\lambda
$$

in which case $P\left(\varepsilon^{\ell_{1}}, \ldots, \varepsilon^{\ell_{m}}\right)=\ell^{m p+(m-1) r} \Phi(\lambda, \ell)^{r} \Delta$, where

$$
\Delta=\prod_{j=1}^{m}\left[\delta\left(\ell_{j_{1}^{\alpha_{1}^{-1}}},-\ell_{j^{\beta_{1}^{-1}}}\right) \cdots \delta\left(\ell_{j^{\alpha_{p}^{-1}}},-\ell_{j_{p}^{\beta_{p}^{-1}}}\right)\right] .
$$

Thus, $\Delta$ is always equal to 0 or 1 . Now it is clear that (regardless of $\alpha_{i}, \beta_{i}$ ) in view of (19), $\Delta$ does not vanish if and only if $\lambda \equiv-\lambda(\bmod \ell)$ or, equivalently,

$$
2 \lambda \equiv 0 \quad(\bmod \ell) .
$$

This equation has only the trivial solution $\lambda \equiv 0(\bmod \ell)$ if $\ell$ is odd, and it has the additional solution $\lambda \equiv \ell / 2(\bmod \ell)$ if $\ell$ is even.

We conclude that

$$
P\left(\varepsilon^{\ell_{1}}, \ldots, \varepsilon^{\ell_{m}}\right)=\ell^{m p+(m-1) r} \Phi(0, \ell)^{r}
$$

if $\ell_{1}=\cdots=\ell_{m}=0$,

$$
P\left(\varepsilon^{\ell_{1}}, \ldots, \varepsilon^{\ell_{m}}\right)=\ell^{m p+(m-1) r} \Phi(\ell / 2, \ell)^{r}
$$

if $\ell$ is even, and $\ell_{1}=\cdots=\ell_{m}=\ell / 2$, and

$$
P\left(\varepsilon^{\ell_{1}}, \ldots, \varepsilon^{\ell_{m}}\right)=0
$$


in all other cases.

As was shown by Hölder,

$$
\Phi(x, n)=\frac{\phi(n)}{\phi\left(\frac{n}{(x, n)}\right)} \mu\left(\frac{n}{(x, n)}\right),
$$

where $\mu(n)$ is the number-theoretic Möbius function [1, p. 164] (cf. [21]). It follows that $\Phi(0, \ell)=\phi(\ell)$ and $\Phi(\ell / 2, \ell)=-\phi(\ell)$.

Substitute these values into the above expressions for $P\left(\varepsilon^{\ell_{1}}, \ldots, \varepsilon^{\ell_{m}}\right)$ and substitute them into (17). Taking into account that $\phi(\ell)^{r}+(-\phi(\ell))^{r}=0$ if $r$ is odd and $\phi(\ell)^{r}+(-\phi(\ell))^{r}=2 \phi(\ell)^{r}$ if $r$ is even, we finally obtain (15).

3. Enumeration. The main result of this paper is the following.

THEOREM 3.1. The number $N_{p, r}(n)$ of nonequivalent $n$-fold coverings of a closed nonorientable surface of genus $p$ with $r \geq 1$ cyclic branch points is expressed by the following formulas:

$$
N_{p, r}(n)= \begin{cases}n^{p-2} \sum_{\substack{\ell \mid n \\ \ell m=n}} \ell^{(m-1) \nu} \phi(\ell)^{r}(2, \ell) \sum_{s=0}^{m-1}[s !(m-s-1) !]^{\nu} & \text { for } r \text { even }, \\ n^{p-2} \sum_{\substack{\ell \mid n \\ \ell m=n}} \ell^{(m-1) \nu} \phi(\ell)^{r} \sum_{s=0}^{m-1}(-1)^{s}[s !(m-s-1) !]^{\nu} & \text { for } r \text { odd, } n \text { odd }, \\ 0 & \text { for } r \text { odd, } n \text { even },\end{cases}
$$

where $\nu:=p-2+r$ is the characteristic of $\mathcal{S}-B, \phi(\ell)$ is the Euler function, and $(2, \ell)$ denotes the greatest common divisor of the numbers 2 and $\ell$.

Proof. Recall that the number of coverings $N_{p, r}(n)$ coincides with the number of orbits of the symmetric group $\mathbf{S}_{n}$ acting by conjugation on the set $\mathfrak{T}_{p, r,(n)^{r}}(n)$. By applying Burnside's lemma we obtain

$$
N_{p, r}(n)=\frac{1}{n !} \sum_{\substack{\ell \mid n \\ \ell m=n}} \frac{n !}{m ! \ell^{m}} \widetilde{T}_{p, r}\left(\ell^{m}\right),
$$

where $\widetilde{T}_{p, r}\left(\ell^{m}\right)$ denotes the number of tuples (1) satisfying (2) and (5) and commuting with a fixed regular permutation $h$ of order $\ell$. As we saw, these are permutation tuples satisfying (in terms of the centralizer $Z(h)$ ) conditions (10)-(13). Since restrictions (10) and (12) are independent of (11) and (13), multiplying the numbers of solutions of both problems, we obtain in the designations adopted above the following proposition.

Proposition 3.2.

$$
\widetilde{T}_{p, r}\left(\ell^{m}\right)=T_{p, r}(m) M_{p, r, m}(\ell) .
$$

Now we make use of formulas (6) and (15). Notice that the last factor in (15) can be represented equivalently as follows:

$$
\left\{\begin{array}{cl}
(2, \ell) & \text { for } r \text { even, } \\
1 & \text { for } r \text { odd, } \ell \text { odd } \\
0 & \text { for } r \text { odd, } \ell \text { even. }
\end{array}\right.
$$


Substituting expressions (25), (6), and (15) (taking into account (26)) into (24), after elementary transformations we obtain the first two formulas (23). Now consider the last case, when $r$ is odd and $n$ is even. According to (15) (or (26)), for even $\ell$ dividing $n$, the factor $M_{p, r, m}(\ell)=0$. Now suppose that $\ell$ is odd. Then $m$ is even. In this case, $\sum_{s=0}^{m-1}(-1)^{s}\left(\begin{array}{c}m-1 \\ s\end{array}\right)^{-\nu}=0$ since $\left(\begin{array}{c}m-1 \\ s\end{array}\right)^{-\nu}=\left(\begin{array}{c}m-1 \\ m-1-s\end{array}\right)^{-\nu}$ and $s$ and $m-1-s$ are of different parity. Thus, $T_{p, r}(m)=0$ and $N_{p, r}(n)=0$.

Remark 1. In our case, the covering surface is nonorientable. Indeed, since the permutation $c_{1}$ is a full cycle, for any permutation $a_{1} \in \mathbf{S}_{n}$ there exists an integer $k$ such that the permutation $a_{1} c_{1}^{k}$ fixes the element 1 . The word $a_{1} c_{1}^{k}$ contains an odd number of letters $a_{j}$; therefore, by the familiar criterion [2], this means that the corresponding covering surface is nonorientable. Besides, by the Riemann-Hurwitz formula it is of characteristic $n \nu$.

Remark 2. It is interesting to compare (23) with the formula for the number $N_{g, r}^{\mathrm{o}}(n)$ of the corresponding coverings of an orientable surface of genus $g$. According to [11] (in a slightly modified form),

$$
N_{g, r}^{\mathrm{o}}(n)=n^{2 g-2} \sum_{\substack{\ell \mid n \\ \ell m=n}} \ell^{(m-1) \nu} \psi(r, \ell) \sum_{s=0}^{m-1}(-1)^{s r}[s !(m-s-1) !]^{\nu},
$$

where $\psi(r, \ell):=\sum_{k=1}^{\ell} \Phi(k, \ell)^{r}$ and $\nu:=2 g-2+r$. At the same time, for the number of the corresponding permutation tuples $T_{g, r}^{\mathrm{o}}(n)$ we conclude from [11] and formula (6) above for $p=2 g$ that

$$
T_{g, r}^{\mathrm{o}}(n)=T_{2 g, r}(n) .
$$

Now let us express $N_{p, r}(n)$ in terms of $T_{p, r}(m), m \mid n$. Formula (6) can be rewritten in the following form:

$$
T_{p, r}(n)=n ! n^{p-2} \sum_{s=0}^{n-1}(-1)^{s r}[s !(n-s-1) !]^{\nu} .
$$

In (23) we can join the first two formulas with the help of the greatest common divisor of three numbers $(2, \ell, r)$. After that, substituting there the right-hand-side expression of $(29)$, we obtain

$$
N_{p, r}(n)= \begin{cases}0 & \text { for } r \text { odd, } n \text { even } \\ n^{p-2} \sum_{\substack{\ell \mid n \\ \ell m=n}} \frac{\ell^{(m-1) \nu} \phi(\ell)^{r}(2, \ell, r)}{m^{p-1}} \frac{T_{p, r}(m)}{(m-1) !} & \text { otherwise. }\end{cases}
$$

For comparison, formula (27) can be rewritten in a similar form as follows:

$$
N_{g, r}^{\mathrm{o}}(n)=n^{2 g-2} \sum_{\substack{\ell \mid n \\ \ell m=n}} \frac{\ell^{(m-1) \nu} \psi(r, \ell)}{m^{2 g-1}} \frac{T_{g, r}^{\mathrm{o}}(m)}{(m-1) !} .
$$

Here are the values of $N_{p, r}(n)$ for $n \leq 7$. For even $r$,

$N_{p, r}(1)=1$,

$N_{p, r}(2)=2^{p}$,

$N_{p, r}(3)=3^{p-2}\left(2^{\nu+1}+1+2^{r}\right)$, 
$N_{p, r}(4)=2 \cdot 4^{p-2}\left(6^{\nu}+3 \cdot 2^{\nu}+2^{r}\right)$,

$N_{p, r}(5)=5^{p-2}\left(2 \cdot 24^{\nu}+2 \cdot 6^{\nu}+4^{\nu}+4^{r}\right)$,

$N_{p, r}(6)=2 \cdot 6^{p-2}\left(120^{\nu}+24^{\nu}+12^{\nu}+3^{\nu} \cdot 2^{r}+2 \cdot 8^{\nu}+4^{\nu}+2^{r}\right)$,

$N_{p, r}(7)=7^{p-2}\left(2 \cdot 720^{\nu}+2 \cdot 120^{\nu}+2 \cdot 48^{\nu}+36^{\nu}+6^{r}\right)$,

and for odd $r$ and odd $n, N_{p, r}(1)=1$,

$N_{p, r}(3)=3^{p-2}\left(2^{\nu+1}-1+2^{r}\right)$,

$N_{p, r}(5)=5^{p-2}\left(2 \cdot 24^{\nu}-2 \cdot 6^{\nu}+4^{\nu}+4^{r}\right)$,

$N_{p, r}(7)=7^{p-2}\left(2 \cdot 720^{\nu}-2 \cdot 120^{\nu}+2 \cdot 48^{\nu}-36^{\nu}+6^{r}\right)$.

3.1. Coverings of the projective plane and the Klein bottle. Consider now the particular cases when $\nu=1$. These are coverings of the projective plane and the Klein bottle with two and one branch points, respectively.

COROLLARY 3.3. The number of nonequivalent $n$-fold coverings of the projective plane with two cyclic branch points is given by the formula

$$
N_{1,2}(n)=\frac{1}{n} \sum_{\substack{\ell \ell n \\ \ell m=n}}(2, \ell) \phi(\ell)^{2} \ell^{m-1} \sum_{s=0}^{m-1} s !(m-s-1) ! .
$$

In particular, if $n=q$ is an odd prime, then

$$
N_{1,2}(q)=\frac{1}{q}\left((q-1)^{2}+\sum_{s=0}^{q-1} s !(q-s-1) !\right) .
$$

The numerical values for $n=1,2,3,4,5,6,7,8$ are $1,2,3,8,16,64,264,1580$.

Formula (32) can be slightly simplified due to the following familiar identity [23] (see also [15] and references therein):

$$
\sum_{s=0}^{n} s !(n-s) !=\frac{(n+1) !}{2^{n}} \sum_{j=0}^{n} \frac{2^{j}}{j+1} .
$$

COROLlary 3.4. The number of nonequivalent n-fold coverings of the Klein bottle with one cyclic branch point is given by the formula

$$
N_{2,1}(n)=2 \sum_{\substack{\ell \mid n \\ \ell m=n}} \frac{m ! \ell^{m-1} \phi(\ell)}{m+1}
$$

if $n$ is odd and $N_{2,1}(n)=0$ if $n$ is even. In particular, if $n=q$ is an odd prime, then $N_{2,1}(q)=q-1+2 q ! /(q+1)$.

Proof. $N_{2,1}(n)$ vanishes for even $n$, and for odd $n$ we should take the second formula of (23) with $\nu=1$. Now for odd $m$, the following elementary identity is valid [19] (see also [22, Example 7.67(c)]):

$$
\sum_{s=0}^{m-1}(-1)^{s}[s !(m-s-1) !]=\frac{2 m !}{m+1} ;
$$

the corollary follows.

The numerical values for $n=1,3,5,7,9,11$ are 1, 5, 44, 1266,72636, 6652810 .

Other numerical data for the projective plane and the Klein bottle are contained in Tables 3.1 and 3.2. 
TABLE 3.1

The number of $n$-sheeted coverings of the projective plane $(p=1)$ with $r$ cyclic branch points.

\begin{tabular}{|l||r|r|r|r|r|r|r|}
\hline$n \backslash r$ & 1 & 2 & 3 & 4 & 5 & 6 & 7 \\
\hline 1 & 1 & 1 & 1 & 1 & 1 & 1 & 1 \\
2 & 0 & 2 & 0 & 2 & 0 & 2 & 0 \\
3 & 1 & 3 & 5 & 11 & 21 & 43 & 85 \\
4 & 0 & 8 & 0 & 128 & 0 & 3968 & 0 \\
5 & 1 & 16 & 232 & 5680 & 132448 & 3189184 & 76426624 \\
6 & 0 & 64 & 0 & 581696 & 0 & 8297164544 & 0 \\
7 & 1 & 264 & 144504 & 107174448 & 76724477856 & 55290551845824 & 39803169903525504 \\
\hline
\end{tabular}

TABLE 3.2

The number of $n$-sheeted coverings of the Klein bottle $(p=2)$ with $r$ cyclic branch points.

\begin{tabular}{|l||r|r|r|r|r|r|}
\hline$n \backslash r$ & 1 & 2 & 3 & 4 & 5 & 6 \\
\hline 1 & 1 & 1 & 1 & 1 & 1 & 1 \\
2 & 0 & 4 & 0 & 4 & 0 & 4 \\
3 & 5 & 13 & 23 & 49 & 95 & 193 \\
4 & 0 & 104 & 0 & 2720 & 0 & 93824 \\
5 & 44 & 1256 & 27344 & 666656 & 15911744 & 382307456 \\
6 & 0 & 30608 & 0 & 415444544 & 0 & 5972357328128 \\
7 & 1266 & 1071540 & 743214744 & 537904137744 & 386934209149536 & 278634137614009920 \\
\hline
\end{tabular}

3.2. Asymptotics. It is evident that in formula (23) for fixed $p$ and $r$ and growing $n$, the term with $\ell=1$ dominates (of course, unless $\nu=0$ or $r$ is odd and $n$ is even). In turn, the dominating terms of its internal sum for $\ell=1$ correspond to $s=0$ and $s=n-1$ and are equal to $(n-1) !^{\nu}$. Therefore we have the next corollary.

COROllary 3.5. Asymptotically for fixed $p$ and $r$ (except for $p=r=1$ ),

$$
N_{p, r}(n) \sim 2 \frac{n !^{\nu}}{n^{r}}=2 n !^{p-2}(n-1) !^{r}
$$

as $n \rightarrow \infty$, where $n$ is odd if $r$ is odd.

By $(6), T_{p, r}(n) \sim 2 n !^{p-1}(n-1) !^{r}$ as $n \rightarrow \infty$, with the same restrictions. So

$$
N_{p, r}(n) \sim \frac{T_{p, r}(n)}{n !} .
$$

Notice that $n !^{p}(n-1) !^{r}$ is the number of tuples (1) satisfying (5). As we see, (2) diminishes this number asymptotically $n ! / 2$ times.

Acknowledgment. The second and third authors are thankful to $\mathrm{Com}^{2} \mathrm{MaC}$ of the Pohang University of Science and Technology for support and hospitality during their visit to Pohang, when the results of the present paper were obtained.

\section{REFERENCES}

[1] T. M. Apostol, Introduction to Analytic Number Theory, Springer, New York, 1976.

[2] C. L. Ezell, Branch point structure of covering maps onto nonorientable surfaces, Trans. Amer. Math. Soc., 243 (1978), pp. 123-133.

[3] G. Frobenius and I. Schur, Ubber die reelen Darstellung der endlichen Gruppen, Sitzer. Königlich Preuss. Akad. Wiss. Berlin, 1906, pp. 186-208.

[4] A. HuRwitz, Über Riemann'sche Flächen mit gegebenen Verzweigungspunkten, Math. Ann., 39 (1891), pp. 1-60.

[5] G. D. James, The Representation Theory of the Symmetric Group, Lecture Notes in Math. 682, Springer, Berlin, 1978. 
[6] G. A. Jones, Enumeration of homomorphisms and surface-coverings, Quart. J. Math. Oxford, 46 (1995), pp. 485-507.

[7] A. Kerber And B. Wagner, Gleichungen in endlichen Gruppen, Arch. Math. (Basel), 35 (1980), pp. 252-262.

[8] J. H. KWAK AND J. LEE, Enumeration of graph coverings, surface branched coverings and related group theory, in Combinatorial Computational Mathematics: Present and Future, S. Hong, J. H. Kwak, et al., eds., Word Scientific, Singapore, 2001, pp. 97-161.

[9] J. H. KwaK, J. Lee, AND A. D. MEDNYKh, Enumerating branched surface coverings from unbranched ones, LMS J. Comput. Math., 6 (2003), pp. 89-104.

[10] J. H. KwaK, J. LEE, AND Y. SHIN, Balanced regular coverings of a signed graph and regular branched orientable surface coverings over a nonorientable surface, Discrete Math., 275 (2004), pp. 177-193.

[11] J. H. KWAK And A. Mednykh, Enumeration of branched coverings of closed surfaces whose branched orders coincide with multiplicity, Studia Sci. Math. Hungarica, to appear; also available online from http://com2mac.postech.ac.kr/papers/2002/02-05.ps.

[12] V. A. Liskovets, Towards the enumeration of subgroups of the free group, Dokl. Akad. Nauk BSSR, 15 (1971), pp. 6-9 (in Russian).

[13] V. Liskovets and A. Mednykh, On the Number of Subgroups in the Fundamental Groups for a Class of Seifert Fibre Spaces, Preprint MATH-AL-15-1997, TU Dresden, Dresden, Germany, 1997.

[14] V. Liskovets And A. Mednykh, The number of subgroups in the fundamental groups of some non-orientable 3-manifolds, in Formal Power Series and Algebraic Combinatorics (Moscow, 2000), D. Krob et al., eds., Springer, Berlin, 2000, pp. 276-287.

[15] T. Mansour, Combinatorial identities and inverse binomial coefficients, Adv. in Appl. Math., 28 (2002), pp. 196-202.

[16] W. S. Massey, Algebraic Topology: An Introduction, Grad. Texts in Math. 56, Springer, New York, 1977.

[17] A. D. Mednykh, On the Hurwitz problem on the number of nonequivalent coverings over a compact Riemann surface, Sibirsk. Mat. Zh., 23 (1982), pp. 155-160, 222 (in Russian); Siber. Math. J., 23 (1983), pp. 415-420 (in English).

[18] A. D. Mednykh, Nonequivalent coverings of Riemann surfaces with a prescribed ramification type, Sibirsk. Mat. Zh., 25 (1984), pp. 120-142 (in Russian); Siber. Math. J., 25 (1984), pp. 606-625 (in English).

[19] A. D. Mednykh, Branched coverings of Riemann surfaces whose branch orders coincide with the multiplicity, Commun. Algebra, 18 (1990), pp. 1517-1533.

[20] A. D. Mednykh and G. G. Pozdnyakova, Number of nonequivalent coverings over a compact nonorientable surface, Sibirsk. Mat. Zh., 27 (1986), pp. 123-131 (in Russian); Siber. Math. J., 27 (1986), pp. 99-106 (in English).

[21] C. A. NicOl AND H. S. VANDIVER, A von Sterneck arithmetical function and restricted partitions with respect to modulus, Proc. Natl. Acad. Sci. USA, 40 (1954), pp. 825-835.

[22] R. P. Stanley, Enumerative Combinatorics, Vol. 2, Cambridge University Press, Cambridge, UK, 1999.

[23] B. SuRY, Sum of the reciprocals of the binomial coefficients, European J. Combin., 14 (1993), pp. 351-353. 